\title{
ON CERTAIN TYPES OF FUNCTIONS VIA GENERALIZED OPEN SETS
}

\author{
BISHWAMBHAR ROY
}

\begin{abstract}
The aim of this paper is to introduce some new classes of functions termed as somewhat $(\mu, \lambda)$-continuous functions, somewhat $(\mu, \lambda)$-open functions and hardly $(\mu, \lambda)$-open functions. Some properties of these newly defined functions are discussed in this paper. Relationships among these functions are also being studied.
\end{abstract}

\section{Introduction}

The concepts of continuous functions and open functions are the most important ideas in the branch of mathematics. In the past few years, mathematicians turned their attention towards the generalization of different open like sets, continuous like functions, and open like functions. In fact, in the last decade, Á. C s á s zár [3]-[5] and many other mathematicians (see [1], 2], 9], [11, [12]) have extensively studied generalized topology and developed theories for this particular branch of mathematics. In this paper, somewhat $(\mu, \lambda)$-continuous, somewhat $(\mu, \lambda)$-open and hardly $(\mu, \lambda)$-open functions are introduced in generalized topological spaces.

Let $\exp X$ denote the power set of a non-empty set $X$. A class $[3] \mu(\subseteq \exp X)$ is called a generalized topology, (briefly, GT) if $\varnothing \in \mu$ and $\mu$ is closed under arbitrary union. The elements of $\mu$ are called $\mu$-open sets and the complements of $\mu$-open sets are known as $\mu$-closed sets. A set $X$ with a GT $\mu$ is known as a generalized topological space (briefly, GTS) and is denoted by $(X, \mu)$.

For any $A \subseteq X$, the generalized $\mu$-closure of $A$ is denoted by $c_{\mu}(A)$ and is defined by $c_{\mu}(A)=\cap\{F: F$ is $\mu$-closed and $A \subseteq F\}$, similarly, $i_{\mu}(A)=$ $\cup\{U: U \subseteq A$ and $U \in \mu\}$ (see [3], [5]). Throughout the paper, $\mu, \lambda$ will always mean GT on the respective sets.

(c) 2016 Mathematical Institute, Slovak Academy of Sciences. 2010 Mathematics Subject Classification: $54 \mathrm{C} 10,54 \mathrm{C} 08$.

Keywords: $\mu$-open set, somewhat $(\mu, \lambda)$-continuity, somewhat $(\mu, \lambda)$-openness.

The author acknowledges the financial support from UGC for the Research Project having grant No. 41-799/2012(SR) UGC, New Delhi. 


\section{Somewhat $(\mu, \lambda)$-continuous functions}

Definition 2.1. A function $f:(X, \mu) \rightarrow(Y, \lambda)$ is said to be somewhat $(\mu, \lambda)$ -continuous if, for each $\lambda$-open set $V$ with $f^{-1}(V) \neq \varnothing$, there is a non-empty $\mu$-open set $U$ such that $U \subseteq f^{-1}(V)$.

Definition 2.2. A function $f:(X, \mu) \rightarrow(Y, \lambda)$ is said to be $(\mu, \lambda)$-continuous [3] if $f^{-1}(V)$ is $\mu$-open in $X$ for each $\lambda$-open set $V$ of $Y$.

\section{Remark 2.3.}

(i) It follows from Definition 2.1 and 2.2 that every $(\mu, \lambda)$-continuous function is somewhat $(\mu, \lambda)$-continuous. The converse is not true (even for topologies) as has been shown in [7].

(ii) If $f:(X, \mu) \rightarrow(Y, \lambda)$ is a somewhat $(\mu, \lambda)$-continuous function and $\nu$ is a GT on $X$ with $\mu \subseteq \nu$, then $f$ is somewhat $(\nu, \lambda)$-continuous.

(iii) If $f:(X, \mu) \rightarrow(Y, \lambda)$ is a somewhat $(\mu, \lambda)$-continuous function and $\eta$ is a GT on $Y$ with $\eta \subseteq \lambda$, then $f$ is somewhat $(\mu, \eta)$-continuous.

Definition 2.4. A subset $A$ of a $\operatorname{GTS}(X, \mu)$ is called $\mu$-dense [6] if $c_{\mu}(A)=X$.

It is easy to observe that $A$ is $\mu$-dense if and only if there is no $\mu$-closed set $C$ in $X$ such that $A \subseteq C \varsubsetneqq X$.

TheOREM 2.5. For a function $f:(X, \mu) \rightarrow(Y, \lambda)$, the followings are equivalent:

(i) $f$ is somewhat $(\mu, \lambda)$-continuous.

(ii) For a $\lambda$-closed set $C$ of $Y$ with $f^{-1}(C) \neq X$, there is a $\mu$-closed subset $F \neq X$ of $X$ such that $f^{-1}(C) \subseteq F$.

(iii) The image of a $\mu$-dense set in $X$ is $\lambda$-dense in $f(X)$.

Proof.

(i) $\Rightarrow$ (ii): Let $C$ be a $\lambda$-closed subset of $Y$ such that $f^{-1}(C) \neq X$. Then $Y \backslash C$ is a $\lambda$-open set in $Y$, where $f^{-1}(Y \backslash C) \neq \varnothing$. By (i), there exists a non-empty $\mu$-open set $U$ in $X$ such that $U \subseteq f^{-1}(Y \backslash C)$. This means that $f^{-1}(C) \subseteq F$ where $F=X \backslash U$ is a $\mu$-closed set in $X$ different from $X$.

(ii) $\Rightarrow$ (i): Let $V$ be a $\lambda$-open set and $f^{-1}(V) \neq \varnothing$. Then $Y \backslash V$ is $\lambda$-closed and $f^{-1}(Y \backslash V)=X \backslash f^{-1}(V) \neq X$. By (ii), there exists a $\mu$-closed set $F \neq X$ of $X$ such that $f^{-1}(Y \backslash V) \subseteq F$. This implies that $X \backslash F \subseteq f^{-1}(V)$ where $X \backslash F$ is non-empty $\mu$-open.

(ii) $\Rightarrow$ (iii): Let $A$ be a $\mu$-dense set in $X$. Suppose that $f(A)$ is not $\lambda$-dense in $f(X)$. Then there exists a $\lambda$-closed set $C$ in $X$ such that $f(A) \subseteq C \varsubsetneqq$ $f(X)$. Clearly, $f^{-1}(C) \neq X$. By (ii), there exists a $\mu$-closed subset $\bar{F}$ in $X$ such that $A \subseteq f^{-1}(C) \subseteq F \varsubsetneqq X$. This is a contradiction to the fact that $A$ is $\mu$-dense in $X$. 


\section{ON CERTAIN TYPES OF FUNCTIONS VIA GENERALIZED OPEN SETS}

(iii) $\Rightarrow$ (ii): Suppose that (ii) is not true. This means that there exists a $\lambda$-closed set $C$ in $Y$ such that $f^{-1}(C) \neq X$ but there is no $\mu$-closed set $F \neq X$ such that $f^{-1}(C) \subseteq F$. This means that $f^{-1}(C)$ is $\mu$-dense in $X$. But by (iii), $f\left(f^{-1}(C)\right)=C$ must be $\lambda$-dense in $Y$, which is a contradiction to the choice of $C$.

Definition 2.6. Two GT's $\mu$ and $\nu$ on $X$ are said to be weakly equivalent if, for each non-empty $\mu$-open set $U$, there exists a nonempty $\nu$-open set $V$ such that $V \subseteq U$, and for each non-empty $\nu$-open set $U$, there exists a non-empty $\mu$-open $V$ such that $V \subseteq U$.

Theorem 2.7. If $f:(X, \mu) \rightarrow(Y, \lambda)$ is a somewhat $(\mu, \lambda)$-continuous function and $\nu$ is a GT on $X$ weakly equivalent with $\mu$, then $f:(X, \nu) \rightarrow(Y, \lambda)$ is somewhat $(\nu, \lambda)$-continuous.

P r o o f. Let $V$ be a $\lambda$-open subset of $Y$ such that $f^{-1}(V) \neq \varnothing$. Since $f:(X, \mu) \rightarrow$ $(Y, \lambda)$ is somewhat $(\mu, \lambda)$-continuous, there exists a nonempty $\mu$-open set $U$ in $X$ such that $U \subseteq f^{-1}(V)$. However, by hypothesis, $\mu$ is weakly equivalent with $\nu$. Therefore, there exists a nonempty $\nu$-open set $U^{*}$ in $X$ such that $U^{*} \subseteq U$. Then $U^{*} \subseteq f^{-1}(V)$. Hence, $f:(X, \nu) \rightarrow(Y, \lambda)$ is somewhat $(\nu, \lambda)$-continuous.

TheOREM 2.8. Let $f:(X, \mu) \rightarrow(Y, \lambda)$ be a somewhat $(\mu, \lambda)$-continuous surjection and $\xi$ be a GT on $Y$ weakly equivalent with $\lambda$. Then $f:(X, \mu) \rightarrow(Y, \xi)$ is somewhat $(\mu, \xi)$-continuous.

Proof. Let $V^{*}$ be a $\xi$-open subset of $Y$ such that $f^{-1}\left(V^{*}\right) \neq \varnothing$. Since $\lambda$ is weakly equivalent with $\xi$, there exists a nonempty $\lambda$-open set $V$ in $Y$ such that $V \subseteq V^{*}$. Now, $\varnothing \neq f^{-1}(V) \subseteq f^{-1}\left(V^{*}\right)$. Since $f:(X, \mu) \rightarrow(Y, \lambda)$ is somewhat $(\mu, \lambda)$-continuous, there exists a nonempty $\mu$-open set $U$ in $X$ such that $U \subseteq f^{-1}(V)$. Then $U \subseteq f^{-1}\left(V^{*}\right)$. Hence, $f:(X, \mu) \rightarrow(Y, \xi)$ is somewhat $(\mu, \xi)$-continuous.

ExAmple 2.9. Let $X=\{a, b, c\}, \mu=\{\varnothing,\{a, b\}, X\}, \lambda=\{\varnothing,\{c\}, X\}$, and $\nu=\{\varnothing,\{c\},\{a, c\}\}$. Then $\mu, \lambda$ and $\nu$ are three GT's on $X$. It can be checked that $\lambda$ and $\nu$ are weakly equivalent. The function $f:(X, \mu) \rightarrow(X, \lambda)$ defined by $f(a)=a, f(b)=b$ and $f(c)=b$ is a surjective, somewhat $(\mu, \lambda)$-continuous, however, $f:(X, \mu) \rightarrow(X, \nu)$ is not somewhat $(\mu, \nu)$-continuous.

Theorem 2.10. If $f:(X, \mu) \rightarrow(Y, \lambda)$ is somewhat $(\mu, \lambda)$-continuous and $g:(Y, \lambda) \rightarrow(Z, \nu)$ is $(\lambda, \nu)$-continuous, then $g \circ f:(X, \mu) \rightarrow(Z, \nu)$ is somewhat $(\mu, \nu)$-continuous.

P r o of. Let $U \in \nu$. Suppose that $(g \circ f)^{-1}(U) \neq \varnothing$. Since $U \in \nu$ and $g$ is $(\mu, \nu)$ -continuous, $g^{-1}(U) \in \lambda$. As $f$ is somewhat $(\mu, \lambda)$-continuous, there exists a non-empty $\mu$-open set $V$ in $X$ such that $V \subseteq f^{-1}\left(g^{-1}(U)\right)$. We have $f^{-1}\left(g^{-1}(U)\right)=$ $(g \circ f)^{-1}(U)$, which implies that $V \subseteq(g \circ f)^{-1}(U)$. Therefore, $g \circ f$ is a somewhat $(\mu, \nu)$-continuous function. 


\section{Somewhat $(\mu, \lambda)$-open and hardly $(\mu, \lambda)$-open functions}

Definition 3.1. A function $f:(X, \mu) \rightarrow(Y, \lambda)$ is said to be somewhat $(\mu, \lambda)$ -open if, for each non-empty $\mu$-open set $U$ in $X$, there exists a non-empty $\lambda$-open set $V$ in $Y$ such that $V \subseteq f(U)$.

Definition 3.2. A function $f:(X, \mu) \rightarrow(Y, \lambda)$ is called $(\mu, \lambda)$-open [8] if $f(U)$ is $\lambda$-open in $Y$ for each $\mu$-open set $U$ in $X$.

According to the two above definitions, it follows that every $(\mu, \lambda)$-open function is somewhat $(\mu, \lambda)$-open, however, as it is well-known, the converse is not true.

TheOREM 3.3. A function $f:(X, \mu) \rightarrow(Y, \lambda)$ is somewhat $(\mu, \lambda)$-open if and only if, for any $A \subseteq X, i_{\mu}(A) \neq \varnothing$ implies that $i_{\lambda}(f(A)) \neq \varnothing$.

P r o o f. It follows from Definition 3.1.

TheOREM 3.4. For a function $f:(X, \mu) \rightarrow(Y, \lambda)$, the followings are equivalent:

(i) $f$ is somewhat $(\mu, \lambda)$-open.

(ii) Inverse image of $\lambda$-dense subset of $Y$ is $\mu$-dense in $X$.

Proof.

(i) $\Rightarrow$ (ii): Suppose that $D$ is a $\lambda$-dense set in $Y$. We need to show that $f^{-1}(D)$ is a $\mu$-dense subset of $X$. Suppose that $f^{-1}(D)$ is not $\mu$-dense in $X$. Then there exists a $\mu$-closed set $B$ in $X$ such that $f^{-1}(D) \subseteq B \varsubsetneqq X$. As $X \backslash B$ is a non-empty $\mu$-open set, by (i), there exists a nonempty $\lambda$-open subset $E$ in $Y$ such that $E \subseteq f(X \backslash B)$. Therefore, $E \subseteq f(X \backslash B) \varsubsetneqq f\left(f^{-1}(Y \backslash D)\right) \subseteq$ $Y \backslash D$. It follows that $D \subseteq Y \backslash E \varsubsetneqq Y$. Thus, $Y \backslash E$ is a $\mu$-closed set and $D \subseteq Y \backslash E \varsubsetneqq Y$. This implies that $D$ is not a $\lambda$-dense set in $Y$, which is a contradiction. Therefore, $f^{-1}(D)$ is a $\mu$-dense subset of $X$.

(ii) $\Rightarrow$ (i): Suppose that $U$ is a nonempty $\mu$-open subset of $X$. We need to show that $i_{\lambda}(f(U)) \neq \varnothing$. If possible, let $i_{\lambda}(f(U))=\varnothing$. Then $c_{\lambda}(Y \backslash f(U))=Y$. Thus, $Y \backslash f(U)$ is $\lambda$-dense in $Y$. Therefore, by (ii), $f^{-1}(Y \backslash f(U))$ is $\mu$-dense in $X$. But, $f^{-1}(Y \backslash f(U)) \subseteq X \backslash U$. Now, $X \backslash U$ is $\mu$-closed. Thus, $X=c_{\mu}\left(f^{-1}(Y \backslash f(U))\right) \subseteq X \backslash U$. This implies that $U=\varnothing$ which is a contradiction to the fact that $U$ is non-empty. Therefore, $i_{\lambda}(f(U)) \neq \varnothing$. This proves that $f$ is somewhat $(\mu, \lambda)$-open.

TheOREM 3.5. For a bijective function $f:(X, \mu) \rightarrow(Y, \lambda)$, the followings are equivalent:

(i) $f$ is somewhat $(\mu, \lambda)$-open.

(ii) For a $\mu$-closed subset $C$ of $X$ with $f(C) \neq Y$, there is a $\lambda$-closed subset $F \varsubsetneqq Y$ such that $f(C) \subseteq F$. 


\section{ON CERTAIN TYPES OF FUNCTIONS VIA GENERALIZED OPEN SETS}

Proof.

(i) $\Rightarrow$ (ii): Let $C$ be any $\mu$-closed subset of $X$ such that $f(C) \neq Y$. Then $X \backslash C$ is a $\mu$-open set in $X$ and $X \backslash C \neq \varnothing$. Since $f$ is somewhat $(\mu, \lambda)$-open, there exists a non-empty $\lambda$-open set $V$ in $Y$ such that $V \subseteq f(X \backslash C)$. Put $F=Y \backslash V$. Clearly, $F$ is $\lambda$-closed in $Y$ and $F \neq Y$. Since, $V \subseteq f(X \backslash C)$, $f(C)=(Y \backslash f(X \backslash C)) \subseteq Y \backslash V=F$.

(ii) $\Rightarrow$ (i): Let $U$ be any nonempty $\mu$-open subset of $X$. Then $C=X \backslash U$ is a $\mu$-closed set in $X$ and $f(X \backslash U)=f(C)=Y \backslash f(U)$ implies that $f(C) \neq Y$. Therefore, by (ii), there is a $\lambda$-closed set $F$ of $Y$ such that $F \neq Y$ and $f(C) \subseteq F$. Clearly, $V=Y \backslash F$ is $\lambda$-open and $V \neq \varnothing$. Also, $V=Y \backslash F \subseteq Y \backslash f(C)=Y \backslash f(X \backslash U)=f(U)$.

TheOREM 3.6. If $f:(X, \mu) \rightarrow(Y, \lambda)$ is a $(\mu, \lambda)$-open map and $g:(Y, \lambda) \rightarrow(Z, \nu)$ is a somewhat $(\lambda, \nu)$-open map, then $g \circ f:(X, \mu) \rightarrow(Z, \nu)$ is a somewhat $(\mu, \nu)$ -open map.

P r o o f. Let $U \in \nu$ and $U \neq \varnothing$. Since $f$ is a $(\mu, \lambda)$-open map, $f(U)$ is $\lambda$-open and $f(U) \neq \varnothing$. Since $g$ is a somewhat $(\lambda, \nu)$-open map and $f(U) \in \lambda$ with $f(U) \neq \varnothing$, there exists a $\nu$-open set $V$ such that $V \subseteq g(f(U))$, which implies that $g \circ f$ is a somewhat $(\mu, \nu)$-open function.

Definition 3.7. A function $f:(X, \mu) \rightarrow(Y, \lambda)$ is said to be hardly $(\mu, \lambda)$-open if, for each $\lambda$-dense subset $A$ of $Y$ that is contained in a proper $\lambda$-open set, $f^{-1}(A)$ is $\mu$-dense in $X$.

From Theorem 3.4 and Definition 3.7, it follows that every somewhat $(\mu, \lambda)$ -open function is hardly $(\mu, \lambda)$-open. The converse is false as shown in the next example.

EXAmple 3.8. Let $X=\{a, b, c\}, \mu=\{\varnothing,\{a\},\{c\},\{a, c\}, X\}$ and $\lambda=\{\varnothing,\{c\}$, $\{a, c\},\{a, b\}, X\}$. Then $\mu$ and $\lambda$ are two GT's on $X$. It can be checked that the identity mapping $f:(X, \mu) \rightarrow(X, \lambda)$ is hardly $(\mu, \lambda)$-open but not somewhat $(\mu, \lambda)$-open.

Theorem 3.9. A function $f:(X, \mu) \rightarrow(Y, \lambda)$ is hardly $(\mu, \lambda)$-open if and only if, for each set $A \subseteq Y$ containing a non-empty $\lambda$-closed set with $i_{\lambda}(A)=\varnothing$, we have $i_{\mu}\left(f^{-1}(A)\right)=\varnothing$.

P r o of. First, assume that $f$ is hardly $(\mu, \lambda)$-open. Let $A \subseteq Y$ be such that $i_{\lambda}(A)=\varnothing$ and let $F$ be a nonempty $\lambda$-closed set contained in $A$. Since, $i_{\lambda}(A)=\varnothing$, $Y \backslash A$ is $\lambda$-dense in $Y$. As $F \subseteq A, Y \backslash A \subseteq Y \backslash F \neq Y, f^{-1}(Y \backslash A)$ is $\mu$-dense in $X$. Thus, $X=c_{\mu}\left(f^{-1}(Y \backslash A)\right)=c_{\mu}\left(X \backslash f^{-1}(A)\right)=X \backslash i_{\mu}\left(f^{-1}(A)\right)$ which implies that $i_{\mu}\left(f^{-1}(A)\right)=\varnothing$. Conversely, assume that $i_{\mu}\left(f^{-1}(A)\right)=\varnothing$ for every $A \subseteq Y$ having the property that $i_{\mu}(A)=\varnothing$, and $A$ contains a nonempty $\lambda$-closed set. 


\section{BISHWAMBHAR ROY}

Let $A$ be a $\lambda$-dense subset of $Y$ that is contained in the proper $\lambda$-open set $U$. Then $i_{\lambda}(Y \backslash A)=\varnothing$ and $\varnothing \neq Y \backslash U \subseteq Y \backslash A$. Thus, $Y \backslash A$ contains a nonempty $\lambda$-closed set, and hence, $i_{\mu}\left(f^{-1}(Y \backslash A)\right)=\varnothing$. Then $\varnothing=i_{\mu}\left(f^{-1}(Y \backslash A)\right)=$ $i_{\mu}\left(X \backslash f^{-1}(A)\right)=X \backslash c_{\mu}\left(f^{-1}(A)\right)$, and hence, $f^{-1}(A)$ is $\mu$-dense in $X$.

TheOREM 3.10. Let $f:(X, \mu) \rightarrow(Y, \lambda)$ be a function. If $i_{\lambda}(f(A)) \neq \varnothing$ for every $A \subseteq X$ having the property that $i_{\mu}(A) \neq \varnothing$ and if there exists a nonempty $\lambda$-closed set $F$ for which $f^{-1}(F) \subseteq A$, then $f$ is hardly $(\mu, \lambda)$-open.

Proof. Let $B \subseteq U \varsubsetneqq Y$, where $B$ is $\lambda$-dense and $U$ is a $\lambda$-open set. Let $A=f^{-1}(Y \backslash B)$ and $F=Y \backslash U$. Obviously, $f^{-1}(F)=f^{-1}(Y \backslash U) \subseteq f^{-1}(Y \backslash B)=A$. Also, $i_{\lambda}(f(A))=i_{\lambda}\left(f\left(f^{-1}(Y \backslash B)\right)\right) \subseteq i_{\lambda}(Y \backslash B)=\varnothing$. Therefore, we must have $\varnothing=i_{\mu}(A)=i_{\mu}\left(f^{-1}(Y \backslash B)\right)=i_{\mu}\left(X \backslash f^{-1}(B)\right)$ which implies that $f^{-1}(B)$ is $\mu$-dense. Hence, $f$ is hardly $(\mu, \lambda)$-open.

TheOREM 3.11. If $f:(X, \mu) \rightarrow(Y, \lambda)$ is hardly $(\mu, \lambda)$-open, then $i_{\lambda}(f(A)) \neq \varnothing$, for every $A \subseteq X$ having the property that $i_{\mu}(A) \neq \varnothing$ and $f(A)$, contains a nonempty $\lambda$-closed set.

P r o o f. Let $A \subseteq X$ be such that $i_{\mu}(A) \neq \varnothing$ and let $F$ be a nonempty $\lambda$-closed set for which $F \subseteq f(A)$. Suppose that $i_{\lambda}(f(A))=\varnothing$. Then $Y \backslash f(A)$ is $\lambda$-dense in $Y$ and $Y \backslash f(A) \subseteq Y \backslash F$ where $Y \backslash F$ is a proper $\lambda$-open set. Since $f$ is hardly $(\mu, \lambda)$-open, $f^{-1}(Y \backslash \bar{f}(A))$ is $\mu$-dense in $X$. But, $f^{-1}(Y \backslash f(A))=X \backslash f^{-1}(f(A))$, and hence, $i_{\mu}\left(f^{-1}(f(A))\right)=\varnothing$. It follows that $i_{\mu}(A)=\varnothing$ which is a contradiction.

The converses of Theorems 3.10 and 3.11 are true provided that $f$ is surjective. Thus, we have the following characterizations for surjective hardly $(\mu, \lambda)$-open functions.

TheOREM 3.12. If $f:(X, \mu) \rightarrow(Y, \lambda)$ is surjective, then the following conditions are equivalent:

(i) $f$ is hardly $(\mu, \lambda)$-open.

(ii) $i_{\lambda}(f(A)) \neq \varnothing$ for every $A \subseteq X$ having the property that $i_{\mu}(A) \neq \varnothing$ and there exists a nonempty $\lambda$-closed set $F \subseteq Y$ such that $F \subseteq f(A)$.

(iii) $i_{\lambda}(f(A)) \neq \varnothing$ for every $A \subseteq X$ having the property that $i_{\mu}(A) \neq \varnothing$ and there exists a nonempty $\lambda$-closed set $F \subseteq Y$ such that $f^{-1}(F) \subseteq A$. Proof.

(i) $\Rightarrow$ (ii) : This is proved in Theorem 3.11.

(ii) $\Rightarrow$ (iii) : Since $f$ is surjective, $F \subseteq f(A)$ implies that $f^{-1}(F) \subseteq A$.

(iii) $\Rightarrow$ (i) : This is proved in Theorem 3.10 .

Theorem 3.13. Let $f:(X, \mu) \rightarrow(Y, \lambda)$ be a hardly $(\mu, \lambda)$-open bijection and $H$ be a $\mu$-closed subset of $X$ such that $f(H)$ is contained in a proper $\lambda$-open set in $Y$. Then $f(H)$ is contained in a proper $\lambda$-closed set in $Y$. 


\section{ON CERTAIN TYPES OF FUNCTIONS VIA GENERALIZED OPEN SETS}

P r o of. Let $H$ be a $\mu$-closed subset of $X$ and $U(\neq Y)$ be a $\lambda$-open subset of $Y$ with $f(H) \subseteq U$. Then $\varnothing \neq Y \backslash U \subseteq f(X \backslash H)$. Thus, $f(X \backslash H)$ contains a non-empty $\lambda$-closed set of $Y$. So, by Theorem 3.12, $i_{\lambda}(f(X \backslash H)) \neq \varnothing\left(\right.$ as $\left.i_{\mu}(X \backslash H) \neq \varnothing\right)$. Hence, by the injectiveness of $f, f(H) \subseteq Y \backslash f(X \backslash H) \subseteq Y \backslash i_{\lambda}(f(X \backslash H))$. Thus, $f(H)$ is contained in a $\lambda$-closed set.

The bijection in the above theorem is essential as the following examples show.

EXAMPLE 3.14.

(a) Let $X=\{a, b\}, \mu=\{\varnothing,\{b\}, X\}, Y=\{a, b, c\}, \lambda=\{\varnothing,\{b, c\},\{a, c\}, Y\}$. Then $\mu$ and $\lambda$ are two GT's on $X$ and $Y$, respectively. Let $f:(X, \mu) \rightarrow$ $(Y, \lambda)$ be defined by $f(a)=a$ and $f(b)=b$. It can be checked that $f$ is hardly $(\mu, \lambda)$-open injection, however, if $H=\{a\}$, then $f(H)$ is not contained in any proper $\lambda$-closed set in $Y$.

(b) Let $X=\{a, b, c, d\}, \mu=\{\varnothing,\{c\},\{a, c\},\{a, b\},\{a, b, c\}, X\}, Y=\{a, b, c\}$, $\lambda=\{\varnothing,\{c\},\{a, c\},\{a, b\}, Y\}$. Then $\mu$ and $\lambda$ are two GT's on $X$ and $Y$, respectively. Let $f:(X, \mu) \rightarrow(Y, \lambda)$ be defined by $f(a)=a f(b)=b$, $f(c)=c, f(d)=a$. It can be checked that $f$ is hardly $(\mu, \lambda)$-open surjection, however, if $H=\{b, d\}$, then $f(H)$ is not contained in any proper $\lambda$-closed set in $Y$.

Definition 3.15. Let $(X, \mu)$ be a GTS. Then a subset $A$ of $X$ is called a $\mu$-generalized closed set (or, for short, $\mu g$-closed set) [10] if and only if $c_{\mu}(A) \subseteq U$ whenever $A \subseteq U$ where $U$ is $\mu$-open in $X$. A GTS $(X, \mu)$ is called $\mu-T_{1 / 2}$ if and only if every $\mu g$-closed set is $\mu$-closed.

LEMMA 3.16. A GTS $(X, \mu)$ is $\mu-T_{1 / 2}$ if and only if, for each $x \in X,\{x\}$ is either $\mu$-open or $\mu$-closed.

Proof. Suppose that $X$ is $\mu-T_{1 / 2}$ and for some $x \in X,\{x\}$ is not $\mu$-closed. Then $X \backslash\{x\}$ is contained in the $\mu$-open set $X$ at most (in case if $X \in \mu$ ). Thus, $X \backslash\{x\}$ is $\mu g$-closed and hence $\mu$-closed. Hence, $\{x\}$ is $\mu$-open.

Let $A$ be any $\mu g$-closed subset of $X$ and $x \in c_{\mu}(A)$. We have to show that $x \in A$. If $\{x\}$ is $\mu$-closed and $x \notin A$, then $x \in c_{\mu}(A) \backslash A$. Thus, $c_{\mu}(A) \backslash A$ contains a non-empty $\mu$-closed set $\{x\}$, a contradiction to [10, Theorem 2.5]. So, $x \in A$. Again, if $\{x\}$ is $\mu$-open then, since $x \in c_{\mu}(A)$, it follows that $x \in A$. Hence, in both the cases, $x \in A$. Thus, $A$ is $\mu$-closed.

Theorem 3.17. Let $(Y, \lambda)$ be $\lambda-T_{1 / 2}$. Then a mapping $f:(X, \mu) \rightarrow(Y, \lambda)$ is hardly $(\mu, \lambda)$-open if and only if it is somewhat $(\mu, \lambda)$-open.

Proof. If $f$ is somewhat $(\mu, \lambda)$-open, then it is hardly $(\mu, \lambda)$-open. Let $f:(X, \mu) \rightarrow(Y, \lambda)$ be hardly $(\mu, \lambda)$-open. Let $A$ be any $\lambda$-dense subset of $Y$. 


\section{BISHWAMBHAR ROY}

Suppose that $y \in Y \backslash A$. Since $A$ is $\lambda$-dense, $i_{\lambda}(Y \backslash A)=\varnothing$. Thus, $\{y\}$ is not $\lambda$-open. Hence, by Lemma 3.16, $\{y\}$ is $\lambda$-closed (as $Y$ is $\lambda$ - $T_{1 / 2}$ ). Therefore, $A$ is contained in the proper $\lambda$-open subset $Y \backslash\{y\}$. Since $f$ is hardly $(\mu, \lambda)$-open, $f^{-1}(A)$ is $\mu$-dense in $X$. Thus, by Theorem 3.4, $f$ is somewhat $(\mu, \lambda)$-open.

Acknowledgements. The author is thankful to the referee for some comments to improve the paper.

\section{REFERENCES}

[1] BAYHAN, S.-KANIBIR, A.-REILLY, I. L.: On decompositions of generalized continuity, Tatra Mt. Math. Publ. 58 (2014), 37-45.

[2] BORSÍK, J.: Generalized oscillations for generalized continuities, Tatra Mt. Math. Publ. 49 (2011), 119-125.

[3] CSÁSZÁR, Á.: Generalized topology, generalized continuity, Acta Math. Hungar. 96 (2002), 351-357.

[4] CSÁSZÁR, Á.: Generalized open sets in generalized topologies, Acta Math. Hungar. 106 (2005), 53-66.

[5] CSÁSZÁR, Á.: $\delta$ - and $\theta$-modifications of generalized topologies, Acta Math. Hungar. 120 (2008), 275-279.

[6] EKICI, E.: Generalized hyperconnectedness, Acta Math. Hungar. 133 (2011), 140-147.

[7] GENTRY, K. R.-HOYLE, H. B.: Somewhat continuous functions, Czechoslovak Math. J. 21 (1971), 5-12.

[8] MIN, W. K.: Some results on generalized topological spaces and generalized systems, Acta Math. Hungar. 108 (2005), 171-181.

[9] PAVLOVIĆ, V.-CVETKOVIĆ, A. S.: On generalized topologies arising from mappings, Bull. Iranian Math. Soc. 38 (2012), 553-565.

[10] ROY, B.: On a type of generalized open sets, Appl. Gen. Topol. 12 (2011), 163-173.

[11] ROY, B.: On weakly ( $\mu, \lambda)$-open functions, Ukrainian Math. J. 66 (2015), 1595-1602.

[12] SHEN, R. X.: A note on generalized connectedness, Acta Math. Hungar. 122 (2009), $231-235$.

Received November 11, 2015

Department of Mathematics
Women's Christian College
6, Greek Church Row
Kolkata-700 026
INDIA
E-mail: bishwambhar_roy@yahoo.co.in 